\title{
Addendum to: Commensurations of the Johnson kernel
}

\author{
TARA E BRENDLE \\ DAN MARGALIT
}

Let $\mathcal{K}(S)$ be the subgroup of the extended mapping class group, $\operatorname{Mod}(S)$, generated by Dehn twists about separating curves. In our earlier paper, we showed that $\operatorname{Comm}(\mathcal{K}(S)) \cong \operatorname{Aut}(\mathcal{K}(S)) \cong \operatorname{Mod}(S)$ when $S$ is a closed, connected, orientable surface of genus $g \geq 4$. By modifying our original proof, we show that the same result holds for $g \geq 3$, thus confirming Farb's conjecture in all cases (the statement is not true for $g \leq 2$ ).

20F36

The purpose of this note is to extend the results of our paper "Commensurations of the Johnson kernel" to the lone remaining case. We briefly review the notation and basic ideas before explaining the improvement. We refer the reader to our earlier paper for further details [1].

Let $S=S_{g}$ denote a closed, connected, orientable surface of genus $g$, and let $\operatorname{Mod}(S)$ denote the extended mapping class group (orientation reversing elements are allowed). The Torelli group $\mathcal{I}(S)$ is the subgroup of $\operatorname{Mod}(S)$ consisting of elements that act trivially on $H_{1}(S, \mathbb{Z})$, and the Johnson kernel $\mathcal{K}(S)$ is the subgroup of $\mathcal{I}(S)$ generated by Dehn twists about separating curves.

The abstract commensurator of a group $\Gamma$, denoted $\operatorname{Comm}(\Gamma)$, is the group of isomorphisms of finite index subgroups of $\Gamma$ (under composition), with two such isomorphisms equivalent if they agree on a finite index subgroup of $\Gamma$. The product of $\phi: G \rightarrow H$ with $\psi: G^{\prime} \rightarrow H^{\prime}$ is a map defined on $\phi^{-1}\left(H \cap G^{\prime}\right)$.

We have the following theorem, which confirms a conjecture of Farb in all cases [2]. In the original paper, we stated and proved the theorem for $g \geq 4$.

Theorem 1 Let $g \geq 3$, and let $G$ be either $\mathcal{I}\left(S_{g}\right)$ or $\mathcal{K}\left(S_{g}\right)$. We have

$$
\operatorname{Comm}(G) \cong \operatorname{Aut}(G) \cong \operatorname{Mod}\left(S_{g}\right)
$$

For $g \geq 5$ and $G=\mathcal{I}\left(S_{g}\right)$, Theorem 1 is due to Farb-Ivanov [3]. McCarthy-Vautaw proved that $\operatorname{Aut}\left(\mathcal{I}\left(S_{g}\right)\right) \cong \operatorname{Mod}\left(S_{g}\right)$ for $g \geq 3$ [5]. Mess proved that $\mathcal{I}\left(S_{2}\right)=\mathcal{K}\left(S_{2}\right)$ 
is an infinitely generated free group, so Theorem 1 certainly does not hold in this case [6]. Also, it is a theorem of Dehn that $\mathcal{I}\left(S_{1}\right)=1$.

Theorem 1 is a consequence of the following more general theorem.

Theorem 2 Let $g \geq 3$, let $H$ be a finite index subgroup of either $\mathcal{I}\left(S_{g}\right)$ or $\mathcal{K}\left(S_{g}\right)$. Any injective homomorphism $\phi: H \rightarrow \mathcal{I}\left(S_{g}\right)$ is induced by an element $f$ of $\operatorname{Mod}\left(S_{g}\right)$ in the sense that $\phi(h)=f h f^{-1}$ for all $h \in H$.

Theorem 2 has various other corollaries. In particular, it follows that finite index subgroups of $\mathcal{I}(S)$ and $\mathcal{K}(S)$ are co-Hopfian, and that finite index subgroups of $\mathcal{I}(S)$ and $\mathcal{K}(S)$ are characteristic in $\mathcal{I}(S)$ up to conjugacy.

Our basic method, following Ivanov, is to translate Theorem 2 into a question about curve complexes. The complex of curves $\mathcal{C}(S)$ is the complex with vertices for isotopy classes of simple closed curves in $S$ and simplices for disjointness. The complex of separating curves $\mathcal{C}_{S}(S)$ is the subcomplex spanned by the separating curves. Finally, the Torelli complex $\mathcal{T}(S)$ has vertices for isotopy classes of separating curves and isotopy classes of bounding pairs in $S$, and simplices for disjointness.

A superinjective map from one curve complex to another is a map that preserves disjointness and nondisjointness (superinjective maps are easily seen to be simplicial and injective). Theorem 2 reduces to the following theorem.

Theorem 3 Let $g \geq 3$. Every superinjective map $\mathcal{C}_{s}\left(S_{g}\right) \rightarrow \mathcal{T}\left(S_{g}\right)$ is induced by an element of $\operatorname{Mod}\left(S_{g}\right)$.

Let $S=S_{g}$, and let $\phi_{\star}: \mathcal{C}_{S}(S) \rightarrow \mathcal{T}(S)$ be a superinjective map; in our original paper, $\phi_{\star}$ is induced by an injective homomorphism $\phi: H \rightarrow \mathcal{I}(S)$, where $H$ is a finite index subgroup of either $\mathcal{I}(S)$ or $\mathcal{K}(S)$. The goal is to show that $\phi_{\star}$ is induced by an element $f$ of $\operatorname{Mod}(S)$. It follows that $f$ induces $\phi$, which gives Theorem 2 .

A key idea for the argument in our original paper is that of a sharing pair. Suppose that $a$ and $b$ are separating curves in $S$ that bound genus 1 subsurfaces $S_{a}$ and $S_{b}$ of $S$, respectively. We say that $a$ and $b$ form a sharing pair for the curve $\beta$ if $S-\left(S_{a} \cup S_{b}\right)$ is connected, and $S_{a} \cap S_{b}$ is an annulus that contains the curve $\beta$. Note that $\beta$ is necessarily nonseparating. The point is, if a map $\phi_{\star}: \mathcal{C}_{S}(S) \rightarrow \mathcal{C}_{S}(S)$ preserves sharing pairs, then we can extend $\phi_{\star}$ to a map $\mathcal{C}(S) \rightarrow \mathcal{C}(S)$ : the nonseparating curve shared by $a$ and $b$ maps to the nonseparating curve shared by $\phi_{\star}(a)$ and $\phi_{\star}(b)$.

The basic outline of the proof of Theorem 3 is as follows.

(1) The image of $\phi_{\star}$ lies in $\mathcal{C}_{S}(S)$. 
(2) The map $\phi_{\star}$ preserves topological types of curves, and it remembers when two curves are on the same side of a third curve.

(3) The map $\phi_{\star}$ preserves sharing pairs.

(4) $\phi_{\star}$ induces a well-defined superinjective map $\hat{\phi}_{\star}: \mathcal{C}(S) \rightarrow \mathcal{C}(S)$.

(5) By a theorem of Irmak, $\hat{\phi}_{\star}$, hence $\phi_{\star}$, is induced by some $f \in \operatorname{Mod}(S)$ [4].

All of the arguments in the original paper are valid in the case of genus 3 except the argument for Step 3. In the remainder of this addendum, we explain how to modify the proof of this step.

Step 3 above is Proposition 4.2 of the original paper, which is a straightforward consequence of Steps 1 and 2 and of the following lemma, which is Lemma 4.1 in the original paper. We restate the lemma (with the case $g=3$ added) and explain how to modify the proof.

Lemma 4 Let $g \geq 3$, and let $a$ and $b$ be curves in $S=S_{g}$ that bound a genus 1 subsurface of $S$. Then $a$ and $b$ are a sharing pair if and only if there exist separating curves $w, x, y$, and $z$ in $S$ with the following properties.

(1) $z$ bounds a genus 2 subsurface $S_{z}$ of $S$

(2) $a$ and $b$ are in $S_{z}$ and intersect each other

(3) $x$ and $y$ are disjoint

(4) $w$ intersects $z$, but not $a$ and not $b$

(5) $x$ intersects $a$ and $z$, but not $b$

(6) $y$ intersects $b$ and $z$, but not $a$

Note that we do not specify whether or not $w$ intersects $x$ or $y$.

One direction of the proof of Lemma 4 works as stated in the original paper for $g \geq 3$. That is, if there exist curves $w, x, y$, and $z$ with the given properties, then $a$ and $b$ form a sharing pair.

It remains to show that, if $a$ and $b$ form a sharing pair in $S_{g}$ for $g \geq 3$, then we can find curves $w, x, y$, and $z$ that satisfy the conditions of the lemma. The idea from the original paper is shown in Figure 1. The reader will notice that this construction is rather complicated, and does not give a useful configuration in $S_{3}$ in any obvious way.

The new idea is to give a simpler configuration that works for every genus $g \geq 3$. This new configuration is shown in Figure 2. 


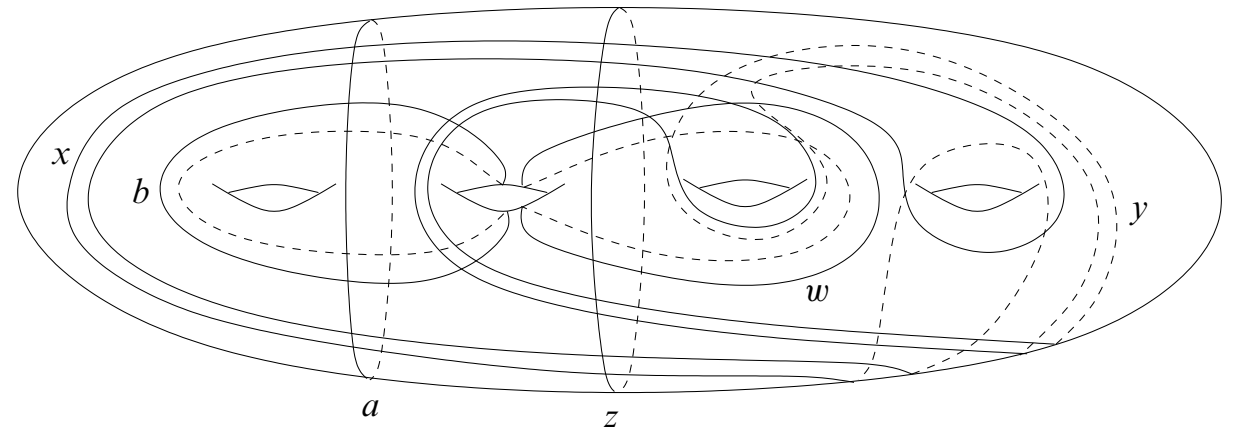

Figure 1: The original construction of the curves $w, x, y$, and $z$.

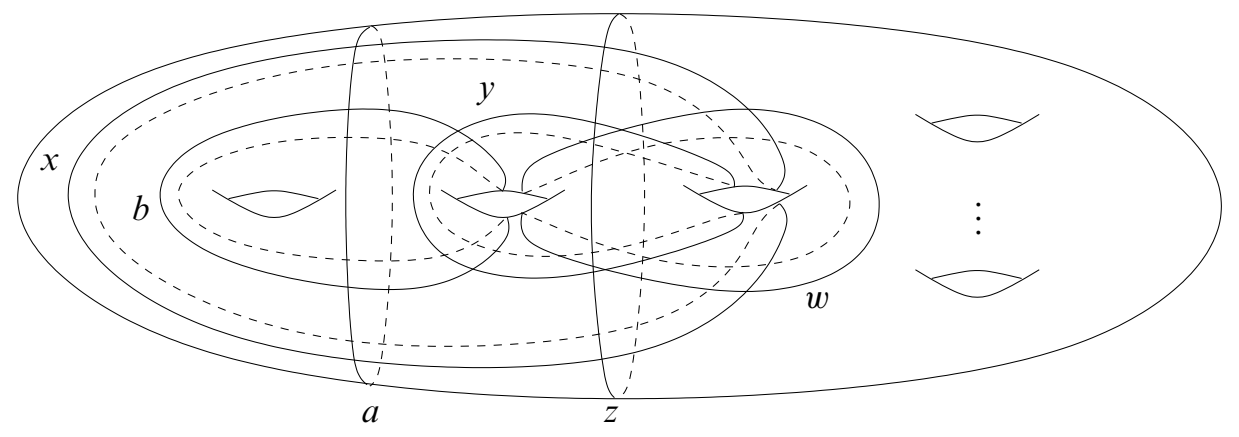

Figure 2: The new construction of the curves $w, x, y$, and $z$.

We remark that the configurations in Figure 1 and Figure 2 are different in an essential way. For instance, consider the intersection of the genus 1 subsurface bounded by $y$ and the genus 2 subsurface bounded by $z$; in Figure 1, this intersection is a disk, whereas in Figure 2 it is an annulus. Perhaps more to the point, in Figure 1, each of $w$, $x$, and $y$ bounds of a genus 1 subsurface, whereas in Figure 2, the curve $x$ does not bound a genus 1 subsurface when $g \geq 4$.

\section{Acknowledgements}

The first author was supported in part by NSF grant DMS-0606882 and the LSU Council on Research Summer Stipend Program. The second author was partially supported by NSF grant DMS-0707279 and the NSF VIGRE program. 


\section{References}

[1] T E Brendle, D Margalit, Commensurations of the Johnson kernel, Geom. Topol. 8 (2004) 1361-1384 MR2119299

[2] B Farb, Automorphisms of the Torelli group, AMS sectional meeting, Ann Arbor, Michigan, March 1 (2002)

[3] B Farb, N V Ivanov, The Torelli geometry and its applications: research announcement, Math. Res. Lett. 12 (2005) 293-301 MR2150885

[4] E Irmak, Superinjective simplicial maps of complexes of curves and injective homomorphisms of subgroups of mapping class groups, Topology 43 (2004) 513-541 MR2041629

[5] JD McCarthy, WR Vautaw, Automorphisms of Torelli groups arXiv: math.GT/0311250

[6] G Mess, The Torelli groups for genus 2 and 3 surfaces, Topology 31 (1992) 775-790 MR1191379

Department of Mathematics, Louisiana State University,

Baton Rouge LA 70803-4918, USA

Department of Mathematics, University of Utah,

155 S 1400 East, Salt Lake City UT 84112, USA

brendle@math.lsu.edu, margalit@math.utah.edu

Proposed: Walter Neumann

Received: 13 September 2007

Seconded: Joan Birman, Shigeyuki Morita

Accepted: 12 October 2007 